\title{
Nativism and the poverty of the stimulus: A demanding argument for the 'innateness' of language
}

\author{
DOMI DESSAIX
}

\section{Abstract}

This article critiques nativism about language (also called linguistic nativism), the view that language capacities are somehow innate, associated especially with Noam Chomsky (for example, 1980, 1993). The focus of the article is on the so-called 'poverty of the stimulus argument' (PSA), often taken to be the backbone of nativism, which says that children's knowledge of language is underdetermined by their linguistic environment. I first argue for how the nativists' claim should be interpreted - an important task given arguments that the notion of 'innateness' in science is problematic (e.g. Mameli \& Bateson 2006). I then outline and critique one example of a PSA, that of Lidz et al. (2003), applied to English anaphoric 'one'. I show that the study is far from successful in demonstrating a PSA for this feature of language. I also argue that the problems with Lidz et al. (2003) are instructive in showing how difficult it is to empirically substantiate a PSA. The article concludes that nativists about language needs to move away from the PSA and look to alternative means of supporting their thesis.

\section{Introduction}

Is language innate? This question has traditionally divided nativists about language from empiricists, or non-nativists. ${ }^{1}$ Whereas nativists argue that explaining language acquisition requires positing innate knowledge of linguistic principles or content, non-nativists - as the name suggests-disagree. No-one, of course, thinks that learning plays no role in language acquisition or that children bring no biases to the task. ${ }^{2}$ Rather, the debate turns on what kind of knowledge or biases children use to acquire language, over and above what is gleaned from the primary linguistic

\footnotetext{
1 I will use the term 'non-nativists' in this article; as discussed later, critics of nativism typically hold that there are domain general constraints on language learning, which may not be in line with the classical empiricist view, e.g. that of Locke (1690).

2 Throughout the article 'language acquisition' refers to first (child) language acquisition.
} 
data (PLD), that is, the linguistic input available in the environment. The principal argument put forward for nativism, and arguably the central pillar of the position, is the 'poverty of the stimulus' argument (PSA). This is essentially the idea that because some linguistic knowledge possessed by children is underdetermined by the PLD in crucial ways, and yet isn't explicable in terms of general learning mechanisms, linguistic knowledge must be innate. The purpose of this article is twofold: (1) to argue for how the nativists' claim should be understood, in light of critiques of the notion of innateness; and (2) to evaluate PSA as evidence for nativism, via an assessment of Lidz et al. (2003), who claim to empirically demonstrate a PSA for the syntax of English anaphoric 'one'. I hope to convince the reader both that this particular study doesn't demonstrate a PSA, and that the PSA more generally is not presently the best avenue for empirically vindicating nativism.

In section two, I consider what exactly the nativists' claim is, attending to Mameli and Bateson's (2006) critique of the concept of innateness. I argue here that nativism about language should be taken as the view that there exists some genetically encoded, environmentally canalised and language-specific knowledge or bias used by children in language acquisition. In section three I lay out the general form of the PSA, as well as Pullum and Scholz's (2002) helpful analysis of it, then consider how exactly a successful PSA could support nativism. In section four I describe a particular PSA for the syntax of anaphoric 'one' (Lidz, Waxman and Freedman 2003, henceforth LWF). In section five I show why I think this PSA fails. Here I discuss Regier and Gahl's (2004) response, arguing that although their model of how the syntax of anaphoric 'one' can be learnt is strictly false, their objection that LWF don't consider all the evidence children might draw on still stands. Then I present three further problems with the study, concerning (1) the nature of the syntactic rule LWF claim to be innate, (2) a dubitable claim about the relevance of grammatical errors in the input, and (3) an unjustified assumption about the nature of the PLD. In section six I discuss the upshot for the PSA: although this is just one study of one construction, it suggests just how difficult it is to empirically demonstrate a PSA. And yet, contra comments of nativists Berwick et al. (2011), I contend that the piecemeal approach exemplified by LWF is the only viable way to substantiate the PSA. My conclusion in section seven is that nativists' efforts may be better directed at investigating other possible sources of evidence for nativism, at the very least until certain difficult questions can be answered about, for example, the nature of the PLD, and the kinds of evidence children use in the acquisition task.

\section{'Innateness' and the nativist's claim}

Of course, whether or not linguistic knowledge is 'innate' depends on what this term means. Attending to this question is particularly important because the idea that the (folk) concept of innateness is relevant to scientific inquiry has been the 
target of recent critique. For example, Mameli and Bateson (2006, henceforth $\mathrm{M} \& \mathrm{~B}$ ) consider and reject some 25 definitions of innateness as possible contenders for the scientific successor to the folk notion, each involving an element of that concept (e.g. species typicality) that has worked its way into scientific discussion. M\&B's thesis, deftly argued, is that because no definition is such that it is both scientifically useful and overlaps sufficiently with the extension of the folk concept, there is no scientific successor to the folk concept of innateness. This verdict isn't necessarily damning to the nativist, who needn't claim that the property they are ascribing to our language capacities is, in fact, a property latched onto by any folk notion. However, the plethora of possible definitions does suggest that nativists cannot simply draw on the term, but must state their claim in other terms. Here I will argue for taking nativism as a three-part hypothesis, and not couched in terms of 'innateness' at all.

But before setting out this hypothesis, it is instructive to show why we should reject the definition of 'innateness' that $M \& B(2006: 160)$ propose for linguistic nativism, as follows:

(1) [The knowledge of a linguistic principle] is innate if and only if its development doesn't involve the extraction of information from the environment.

$\mathrm{M} \& \mathrm{~B}$ point out that the central argument of nativism, viz. the PSA, is essentially the idea that because the crucial information required to learn certain grammatical rules isn't available in the linguistic environment, children's knowledge of those rules is innate. Therefore, $\mathrm{M} \& \mathrm{~B}$ argue, although nativists often use terms like 'genetic determination' and 'genetic endowment' (Chomsky 1980, 1993), we should understand nativism simply as a claim about the non-environmental source of linguistic knowledge, rather than as a claim about its genetic provenance.

But (1) isn't a plausible characterisation of nativism, for three reasons. Firstly, it would make the nativists' claim unsubstantial, for to say that the relevant knowledge is non-environmental in origin doesn't say anything about where it does come from. In fact, I would suggest that (1) only sounds plausible because it rests on a covert genes-environment distinction; that is, it implies that the relevant information is genetically derived. Secondly, nativists don't think, as is suggested by (1), that environmental information isn't necessary to language acquisition, just that it isn't sufficient. Thirdly, (1) doesn't distinguish nativism from non-nativism: non-nativists don't have to hold that environmental input alone is sufficient for language learning, but rather may (typically do) believe that the acquisition 
of linguistic structures depends on a host of domain-general mechanisms or biases, ${ }^{3}$ which may not themselves have been acquired via extraction of information from the environment. Thus (1) does not characterise the nativists' position.

How then should the innateness claim of nativists be understood? I don't think any single proposal considered by $M \& B$ will do. Rather, I think nativism should be analysed as the conjunction of three claims. Two of these involve notions considered by M\&B: genetic encoding, the idea that a trait is encoded in some (even very large) set of genes; and environmental canalisation, the idea that a trait is insensitive to variation in some range of environmental conditions, but sensitive to variation in others. The third claim is language-specificity, that is, the idea that a principle or bias is specific to the domain of language, not generalisable across cognitive domains. Thus we have:

(2) The nativist's claim: there exists some language-specific, environmentally canalised, and genetically encoded knowledge or bias, used by children in language acquisition.

Note that (2) doesn't contain the term 'innateness' at all. Now I will explain each element in turn, and why it should be considered part of the nativist's claim about language. First, we have language-specificity. This is a crucial element of the nativist's claim. As I have already intimated, this is ultimately what distinguishes the nativist from the non-nativist: only the former has to believe that children have, in addition to domain-general learning mechanisms, some bias or knowledge whose function or content is strictly linguistic. Second, we have environmental canalisation, which must also hold for nativism to be vindicated; according to the nativist, children's acquisition of a certain linguistic principle is insensitive to variation in certain conditions of their linguistic environment, but sensitive to variation in others. In particular, children's acquisition of some linguistic principle $p$ is insensitive to the evidence for $p$ in the PLD; that is, it is environmentally canalised with respect to the amount (accuracy, etc.) of linguistic input relating to $p$. However, the acquisition of $p$ is sensitive to other environmental conditions, notably to the presence of some PLD (i.e. linguistic input is required for children to acquire language normally).

As for the third component of (2), I think it is essential that nativism be committed to something like genetic encoding. Consider that the basic contention of nativists is that children know some linguistic principle without the presence of (sufficient) evidence for that principle in their linguistic environment. If the required information is nowhere in the environment, where else could it plausibly come from, if not from genes? I take it that no-one believes linguistic knowledge is the kind of thing that could be passed on via other biological routes of inheritance,

3 A learning mechanism, bias or item of knowledge is domain-general if it isn't specific to any single cognitive domain (language, numeracy, etc.) but applicable to many, e.g. a domain-general bias towards minimising processing costs (O’Grady 2012). 
for example, along with the transferral of gut flora from mother to infant during labour. Given the lack of plausible alternatives, then, the nativist's claim just has to be that the linguistic knowledge displayed by young learners is encoded genetically. Contra M\&B's suggestion above, then, it strikes me that we should treat the common reference to genes in nativist literature seriously, ${ }^{4}$ as an empirical claim about the provenance of some linguistic knowledge. Of course, this is not to say that the nativist must be committed to the existence of a single language-specific gene, or even small set thereof. But they must think something along these lines: there is some combination of genes such that if they were knocked out or otherwise interfered with, the knowledge of certain linguistic principles would not develop in the individual, and yet other cognitive capacities would remain more or less intact.

The next question to consider is what counts as evidence for (2). The discovery of language-specific genes (in the sense just discussed) would be something of a holy grail for the view. ${ }^{5}$ The existence of language universals may also constitute evidence (provided their universality was not explicable via domain-general considerations), for although the universality of a linguistic principle wouldn't prove that it is genetically encoded, this might be offered as the best explanation of its presence in all human languages. But no kind of evidence for nativism has been as much discussed as the PSA. This argument, and how it is supposed to substantiate nativism, is the subject of the next section.

\section{Evidence for nativism: The PSA}

The PSA has been treated as something of a mainstay for nativism. ${ }^{6}$ What exactly is the argument? Its general form is as follows:

(3) The PSA

For linguistic principle $p$ :

1. At a particular stage of learning, children have acquired $p$

2. The crucial evidence for $p$ is absent from a typical PLD at the age when acquisition of $p$ is demonstrated

3. $p$ is not an instance of a domain-general principle

therefore

\footnotetext{
4 For example, Chomsky (1980), Pinker and Bloom (1990), Hauser et al. (2002).

5 Consider the FOXP2 gene, often called 'the language gene' in articles for the general audience (for example, Trafton 2014). Unfortunately, it looks like this particular gene is involved in both linguistic and non-linguistic capacities (Nudel \& Newbury 2013).

6 For example, Akhtar $(2004,460)$ holds that 'the entire nativist approach relies on the poverty of the stimulus argument'.
} 
4. $p$ is innate, i.e. (conforming to our statement of nativism above) it is languagespecific, genetically encoded, and environmentally canalised.

How would one go about proving (3)? Here Pullum and Scholz's (2002: 19) analysis of the PSA is useful, an adapted version of which is as follows:

(4) A successful PSA must:

i. characterise the acquirendum (describe the linguistic principle that is alleged to be known)

ii. specify the lacuna (identify the evidence that would be required to learn the acquirendum)

iii. show the inaccessibility of the lacuna (provide evidence that learners do not in fact encounter the lacuna)

iv. show the indispensability of the lacuna (show why learning the acquirendum requires exposure to the lacuna)

v. prove acquisition (provide evidence that learners actually know the acquirendum).

This gives us a clear picture of what is required for a PSA to be successfully shown. Before I describe a study that aims to do just this for the syntax of anaphoric 'one', I want to discuss how I think a successful PSA could substantiate the trifold claim in (2). A successful PSA should convince us that a certain linguistic principle is environmentally canalised because it would show us that certain linguistic knowledge acquired by children is insensitive to variation in the presence/absence of the lacuna sentences in their linguistic environment; that is, regardless of whether the lacuna sentences are present in the PLD, the relevant linguistic knowledge is acquired by children of a certain age. A successful PSA should also prove that the principle is language-specific because in order to show that the lacuna sentences are truly indispensable it must be demonstrated that no domain-general principle suffices to explain children's possession of the acquirendum. This in turn lends support to the genetically encoded component of the nativist's claim because, as argued in section 2 above, if children know some language-specific information that isn't contained anywhere in the environment, then genes are the only likely source of this information. ${ }^{7}$ Therefore, an ideal PSA, which furnishes all the elements of (4) above, would be strong support for the nativist view, as specified in (2).

7 Cf. Chomsky's (1980: 9) comment: 'the argument from poverty of the stimulus leaves us no reasonable alternative but to suppose that these properties [of language] are somehow determined in universal grammar, as part of the genotype'. 


\section{A PSA for anaphoric 'one'}

\section{Lidz, Waxman and Freedman (2003)}

LWF claim to have substantiated a PSA for the syntactic properties of English anaphoric 'one'. Although the authors make use of the term 'innateness', nowhere in their paper is the term defined; essentially, we are not told what their study, if successful, demonstrates about anaphoric 'one'. But I think we should take LWF as aiming to support nativism in the way I have just canvassed. Before describing the study, I will illustrate the relevant behaviour of anaphoric 'one'. Consider:

(5) Lillian wore this gold tiara, and Gabby wore that one.

Here 'one' is anaphoric (refers back) to 'gold tiara', not to 'tiara'. Competent speakers know from this utterance that Gabby's tiara was also gold. Thus we see that 'one' refers back to the noun plus any adjectival material present, i.e. to the nominal node $\left(\mathrm{N}^{\prime}\right)$ below, rather than to the bare noun $(\mathrm{N})$ :

(6)

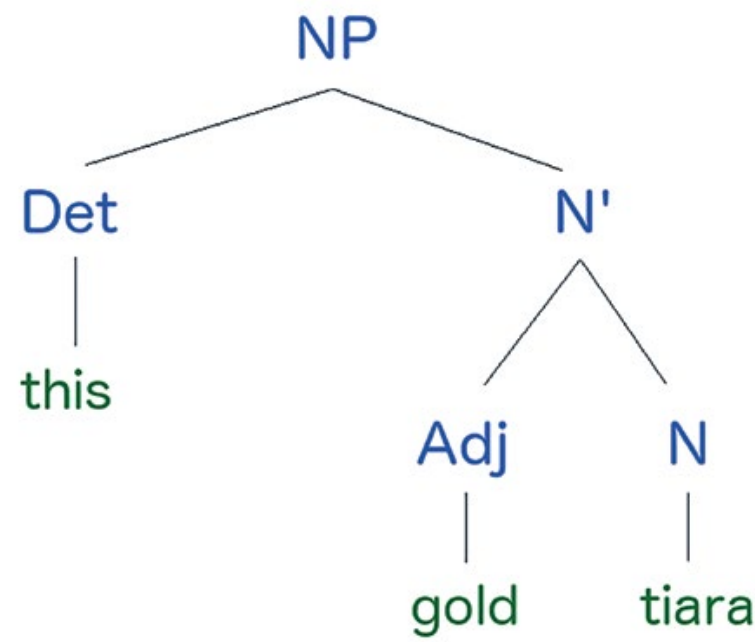

This syntactic fact about what level of the NP 'one' refers to constitutes LWF's acquirendum (4i, above). But note that strings like (5) won't help children to learn the rule: all such sentences confirm both the hypothesis that 'one' refers to [N'] and the hypothesis that 'one' refers to $[\mathrm{N}]$ (every case of a gold tiara is a case of a tiara). Thus, LWF argue, the evidence required to arrive at the correct hypothesis is an utterance like:

(7) Lillian wore a gold tiara, but Gabby didn't wear one 
used in a situation where Gabby is wearing a tiara of another colour, not gold. Such utterances (called Baker events) ${ }^{8}$ constitute the lacuna (4ii). According to LWF, such sentences are indispensable (4iv) because they are the only sentences that rule decisively in favour of the hypothesis 'one' $=[\mathrm{N}$ '], and against 'one' $=[\mathrm{N}]$. As far as the inaccessibility (4iii) of Baker events, LWF's evidence comes from corpora. Analysing data of about 55,000 utterances of child-directed speech, ${ }^{9}$ LWF found that only 0.2 per cent of the cases of anaphoric 'one' were Baker events. LWF also found that 0.5 per cent of cases of anaphoric 'one' were part of ungrammatical sentences. Combining these findings, LWF claim that the crucial evidence is inaccessible: it occurs less often than ungrammatical uses of 'one', therefore to the learner it would be 'indistinguishable from noise in the input' (LWF: B68).

And, finally, what evidence is there that infants have acquired this rule $(4 \mathrm{v})$ ? To demonstrate this, LWF tested the preferential-looking behaviour of 18-monthold infants in a two-phase experiment, best described by the authors themselves:

During the familiarisation phase, an image of a single object (e.g. a yellow bottle) was presented three times [...] accompanied by a recorded voice that named the object with a phrase consisting of a determiner, adjective and noun (e.g. Look! A yellow bottle). During the test phase, two objects appeared simultaneously on opposite sides of the television monitor (e.g. a yellow bottle and a blue bottle). Both objects were from the same category as the familiarisation object, but only one was the same colour. Infants were randomly assigned to one of two conditions which differed only in the linguistic stimulus. In the control condition, subjects heard a neutral phrase (Now look. What do you see now?). In the anaphoric condition, subjects heard a phrase containing the anaphoric expression 'one' (Now look. Do you see another one?) (LWF: B70).

On the assumption that infants prefer a visual stimulus that 'matches' a concurrent linguistic stimulus (e.g. one that can be understood as a referent of one of the words heard), the expectation was that the infants would look longer at the yellow bottle only in the anaphoric condition. This was precisely LWF's finding, from which they infer that the infants have indeed acquired the acquirendum. Thus LWF conclude that they have empirically demonstrated a PSA for the syntax of anaphoric 'one'. 


\section{Criticism of LWF}

Critical responses to LWF dispute both the inaccessibility and indispensability of the evidence, and the infants' acquisition of the acquirendum (Regier \& Gahl 2004, Akhtar et al. 2004, Tomasello 2004). I cannot present and assess all these critiques, so I will canvass just one, that of Regier and Gahl (2004), before discussing three criticisms of my own.

\section{Regier and Gahl (2004)}

Regier and Gahl (2004, henceforth R\&G) dispute the indispensability of the evidence LWF cite for the acquisition of anaphoric 'one'. They point out that LWF's assumption that exposure to Baker events is necessary to acquire the relevant rule amounts to the view that only direct, positive evidence - that is, the presence of certain strings in the linguistic environment-is relevant to language acquisition. But there is no reason, they argue, to rule out the possibility that learners might use indirect, negative evidence - that is the absence of certain strings - to weigh up grammatical hypotheses. R\&G go on to describe a Bayesian model that uses just this kind of evidence to successfully learn the syntactic properties of 'one', without exposure to Baker events. Essentially, this model takes into account the absence of situations in which an utterance like (5) ('Lillian wore this gold tiara, and Gabby wore that one) is used when Gabby is wearing a tiara that isn't gold. The idea behind this is as follows (R\&G: 150). The hypothesis 'one' $=\left[_{\mathrm{N}}\right.$, gold tiara $]$ predicts that the referent of 'one' will be gold, and this prediction will always be confirmed. By contrast, the hypothesis that 'one' $=\left[_{\mathrm{N}}\right.$ tiara $]$ predicts that the referent of 'one' will be a tiara of any colour, so the learner can expect that it will sometimes be used to refer to tiaras that aren't gold. But this will never happen. And this absence constitutes implicit negative evidence against 'one' $=\left[{ }_{\mathrm{N}}\right.$ tiara $]$.

However, as Lidz and Waxman (2004, henceforth L\&W) point out in response, R\&G's model doesn't accurately represent the actual grammar of anaphoric 'one'. It works on the assumption that anaphoric 'one' necessarily takes a nominal phrase $\left(\left[\mathrm{N}^{\prime}\right]\right)$ as its antecedent as long as there is one available. But this is false. Consider:

(8) I like this red ball and you like that one.

On its most felicitous reading, 'one' does refer to 'red ball', i.e. to [N']. But in certain circumstances, 'one' can refer to the bare noun 'ball' (L\&W: 164). For example, imagine you and I must each choose a ball from a selection of various colours before playing a game. If you pick out a yellow ball and I a red one, I might say to you: 'Well, I like this red ball, and you like that one, so let's play' (probably putting stress on 'red'). Yet on R\&G's model such cases are impossible, meaning that a child learner operating on their model would arrive at a false grammar of English. $\mathrm{L} \& \mathrm{~W}$ are right that such examples show that the model is unrealistic (as R\&G 
themselves acknowledge). But this doesn't diminish the general point: learners may come to discriminate among several hypotheses consistent with the same data just by considering negative evidence (R\&G: 153). LWF overlook this possibility, and neither they nor L\&W even attempt to demonstrate that negative evidence is irrelevant to the acquisition of anaphoric 'one'.

Furthermore, I would counter that L\&W's observation that 'one' may have several possible antecedents depending on the context also reveals a problem for LWF, viz. the vagueness in what is being claimed to be innate. It has emerged that anaphoric 'one' only typically refers to the upper [N'] phrase where available, but that sometimes it does not. This changes the acquirendum significantly. What linguistic principle is being claimed to be innate? The possibility of anaphoric 'one' taking a nominal antecedent, depending on the circumstances? This is not just a weak syntactic rule; it is not clearly a rule at all.

\section{Three further problems}

Now I will discuss three further criticisms of LWF. The first relates, like the previous point, to the nature of the knowledge being claimed to be innate. Neither LWF nor L\&W (nor indeed their critics) make any mention of the fact that 'one' is a single anaphoric element of a single language. There is no discussion of how LWF's acquirendum, a syntactic rule governing anaphoric 'one', is supposed to relate to the wider phenomenon of English anaphora, nor to anaphora in the many thousands of languages other than English, in which anaphora may work very differently. This is a problem because even assuming there is a fixed syntactic rule governing the behaviour of anaphoric 'one', it is just not plausible that such a parochial rule, specific to one language, is the kind of principle that could be innate (that is, in terms of (2), genetically encoded and environmentally canalised). I suspect that the authors would respond to this by saying that they aren't actually claiming that a syntactic rule governing just English anaphoric 'one' is innate, but that some more general (but still language-specific, not domain-general) principle of which it is an instance, is innate. But neither LWF nor $\mathrm{L} \& \mathrm{~W}$ make this explicit, let alone try to characterise the more general rule. Without describing what is actually supposed to be innate, LWF's claim is very unclear.

My second worry relates to LWF's argument for the inaccessibility of Baker events. Recall that this argument used the fact that such utterances are rarer in the data (0.2 per cent of all cases of anaphoric 'one') than the grammatically incorrect utterances containing anaphoric 'one' ( 0.5 per cent of all cases of anaphoric 'one') to prove that Baker events are 'indistinguishable from noise in the input' (LWF: B68). Though LWF don't cite a single example of the ungrammatical cases they found, their discussion suggests that they included in that 0.5 per cent every ungrammatical utterance that happened to contain anaphoric 'one'. Yet many of the 
errors in utterances containing anaphoric 'one' will not involve 'one' at all. And I don't see why we should assume that the presence of an error in a sentence in the PLD would lead a learner to disregard the whole sentence as irrelevant 'noise'. Consider that if 60 per cent of that 0.5 per cent of errors didn't involve anaphoric 'one' directly, this reduces the actual (relevant) noise to $<0.2$ per cent, and LWF's argument for the inaccessibility of the evidence fails.

In fact, running my eye over a good section of the data used by LWF, I found no example of an error involving anaphoric 'one' directly. But I did find a handful of ungrammatical utterances containing anaphoric 'one' in which the error is quite clearly irrelevant. Consider the following exchange, in which the mother, partly reproducing the child's utterance, omits the grammatically obligatory subject of 'see'.

(9) CHILD: *see other one put (to)gether.

[...] MOTHER: * see the other one and put them together? ${ }^{10}$

The mother's utterance is ungrammatical, but the error has nothing to do with 'one', let alone its anaphoric properties. Another case, also a repetition of the child's error, involved a similarly irrelevant omission (of the preposition 'for'):

(10) CHILD: *use the other one scissors.

MOTHER: *use the other one scissors.

MOTHER: he means use the other hand for scissors.

(10) is also interesting because the mother's next utterance contains corrective information, potentially accessible to the child (though not directed to him), which might have led him to register his utterance as infelicitous in some way. Now even if neither of these examples were included in LWF's 0.5 per cent, it remains to be seen whether or not the errors they did include were relevant to the acquirendum.

A final disputable assumption of LWF relates to this last point about the possibility of evidence available to a child besides the speech directed to her. Both corpora used by LWF consist of exclusively adult-child linguistic interactions. The idea that only child-directed speech belongs in the PLD amounts to the assumption that children cannot learn from surrounding speech. Yet this idea is not, to my knowledge, backed up by anything more than an intuition about how children learn language. Furthermore, it is questionable on empirical grounds: there exists, for example, some evidence of indirect, unconscious language learning, (Saffran et al. 1997).

10 (9) and (10) are from the Adam corpus (Brown 1973). 


\section{The upshot: Curbing PSA enthusiasm}

What is the upshot of these criticisms for the PSA and nativism more generally? Of course, even if all the criticisms directed at LWF are correct, it is just one study; other PSAs might be successful. But I think the objections raised suggest that proving an instance of a PSA is a difficult business indeed. A few questions suggested by those objections are as follows: (1) do children use negative evidence in language acquisition?; (2) which acquirenda are, and which are not, plausibly 'innate'?; (3) what constitutes noise for the learner?; and (4) does the PLD include surrounding speech? These are all very difficult questions, but all of them, as far as I can see, need to be answered before we can take PSAs seriously.

Interestingly, Berwick et al. (nativists if anyone is) say that they are 'unimpressed' by PSAs in the vein of LWF: that is, those which focus on 'some isolated linguistic phenomenon that children appear to master and conclude that because there is not enough evidence for that phenomenon in isolation, it must be innate' (Perfors et al. 2011, cited in Berwick et al. 2011: 1231). Yet Berwick et al. do not explain how else to proceed with establishing the argument. Instead, they seem to think that to support the PSA it is sufficient to cite certain interesting and general linguistic phenomena, among which are the 'constrained ambiguities' of certain strings. For example, the utterance 'The senator called the donor from Texas' is two ways but not three ways ambiguous: it can mean that the senator called from Texas, or that the donor was from Texas, but not that the senator was from Texas. Yet it isn't clear why such phenomena require a different treatment to LWF's treatment of anaphoric 'one'. The reason for the constrained ambiguity in the cited example is that English NP elements cannot be separated from each other by a verb, thus 'from Texas' cannot be parsed with the 'the senator' and so cannot be interpreted as a property ascribed to him. This is an instance of structure dependency in English. Why wouldn't a PSA for structure dependency require a study of the kind LWF offer for anaphoric 'one'? As Pullum and Scholz's (2002) analysis made clear, it is the nature of the PSA that it makes specific empirical claims both about children's knowledge and about the utterances found in the PLD, neither of which Berwick et al. attempt to substantiate for the case of structure dependency.

In short, contra Berwick et al., I don't see how a compelling PSA could be made proceeding in any other way than the way in which LWF do in fact proceed. Our upshot then is quite grim for the PSA: not only do the many problems with LWF suggest how demanding it is to verify the argument, but it seems that this study represents the only way a PSA can be established. 


\section{Conclusion}

I have critiqued nativism about language, and the foremost argument cited in favour of it, the PSA. I argued for a specific interpretation of the nativist's claim, which doesn't rely on the folk notion of innateness. And, finally, I assessed LWF's attempt at proving a PSA, and found it unsuccessful. PSAs may one day have a greater chance of being empirically substantiated, once we have made headway with some of the difficult questions pointed to in the previous section. Until such time, however, I think the nativist would do well to focus on other possible avenues for substantiating their claim. For example, particular cases of very late first language acquisition (e.g. the interesting case of Chelsea) ${ }^{11}$ could support the existence of genetically encoded, environmentally canalised, language-specific knowledge or capacities. If the debate about linguistic nativism revolved around other evidence besides the PSA, it may prove fruitful for both sides.

\section{Linguistic abbreviations}

Adj $=$ adjective

Det $=$ determiner

$\mathrm{N}=$ noun

$\mathrm{N}^{\prime}=$ nominal phrase

$\mathrm{NP}=$ noun phrase

\section{Bibliography}

Akhtar, N. (2004). 'Nativist Versus Constructivist Goals in Studying Child Language'. Journal of Child Language 31, 459-462. doi.org/10.1017/S0305000904006063

Akhtar, N., Callanan, M., Pullum, G. \& Scholz B. (2004). 'Learning Antecedents for Snaphoric One’. Cognition 93, 141-145. doi.org/10.1016/j. cognition.2003.12.002

Baker, C.L. (1978). Introduction to Generative-Transformational Syntax. Englewood Cliffs, NJ: Prentice-Hall.

Berwick, R., Pietroski, P., Yankama, B. \& Chomsky, N. (2011). 'Poverty of the Stimulus Revisited'. Cognitive Science, 35, 1207-1242. doi.org/10.1111/j.15516709.2011.01189.x

11 Chelsea is a deaf woman who started to learn sign language at the age of 32, and whose linguistic abilities remain far from full competence. Her case is particularly interesting for several reasons; for example, she has an intact number sense (Curtiss 2014). 
Brown, R. (1973). A First Language: The Early Stages. Cambridge, MA: Harvard University Press. doi.org/10.4159/harvard.9780674732469

Chomsky, N. (1980). 'Rules and Representations'. The Behavioural and Brain Sciences, 3, 1-61. doi.org/10.1017/S0140525X00001515

Chomsky, N. (1993). 'On the Nature, Use, and Acquisition of Language'. In A. Goldman (ed.), Readings in Philosophy and Cognitive Science. Cambridge, Mass.: MIT Press.

Curtiss, S. (2014). 'The Case of Chelsea: The Effects of Late Age at Exposure to Language on Language Performance and Evidence for the Modularity of Language and Mind'. In C. T. Schütze \& L. Stockall (eds), Connectedness: Papers by and for Sarah Van Wagenen. UCLA Working Papers in Linguistics 18.

Hauser, M.D., Fitch, W.T. \& Chomsky, N. (2002). 'The Faculty of Language: What Is It, Who Has It, and How Did It Evolve?' Science, 298(5598), 1569-1579. doi. org/10.1126/science.298.5598.1569

Laurence, S. \& Margolis, E. (2001). 'The Poverty of the Stimulus Argument'. The British Journal for the Philosophy of Science, 52(2), 217-276. doi.org/ 10.1093/bjps/52.2.217

Lidz, J. \& Waxman, S. (2004). 'Reaffirming the Poverty of the Stimulus Argument: A Reply to the Replies'. Cognition, 93, 157-165. doi.org/10.1016/j. cognition.2004.02.001

Lidz, J., Waxman, S. \& Freedman, J. (2003). 'What Infants Know about Syntax but Couldn't Have Learned: Experimental Evidence for Syntactic Structure at 18 Months'. Cognition ,89, B65-B73. doi.org/10.1016/S0010-0277(03)00116-1

Locke, J. (1690). An Essay Concerning Human Understanding. Kitchener, Ontario: Batoche Books. doi.org/10.1093/oseo/instance.00018020

Mameli, M. \& Bateson, P. (2006). 'Innateness and the Sciences'. Biology and Philosophy, 21, 155-188. doi.org/10.1007/s10539-005-5144-0

Nudel, R. \& Newbury, D.F. (2013). Wiley Interdisciplinary Reviews: Cognitive Science, 4, 547-560. doi.org/10.1002/wcs. 1247

O'Grady, W. (2012). 'Three Factors in the Design and Acquisition of Language'. Wiley Interdisciplinary Reviews: Cognitive Science, 3, 493-499. doi.org/10.1002/ wcs. 1188

Pinker, S. \& Bloom, P. (1990). 'Natural Language and Natural Selection'. Behavioral and Brain Sciences, 13, 707-784. doi.org/10.1017/S0140525X00081061 
Pullum, G. \& Scholz, B. (2002). 'Empirical Assessment of Stimulus Poverty Arguments'. The Linguistic Review, 19, 9-50.

Regier, T. \& Gahl, S. (2004). 'Learning the Unlearnable: The Role of Missing Evidence’. Cognition, 93, 147-155. doi.org/10.1016/j.cognition.2003.12.003

Saffran, J.R., Newport, E.L., Aslin, R.N., Tunick, R.A. \& Barrueco, S. (1997). 'Incidental Language Learning: Listening (and Learning) Out of the Corner of Your Ear'. Psychological Science, 8(2), 101-105. doi.org/10.1111/j.1467-9280.1997. tb00690.x

Suppes, P. (1974). 'The Semantics of Children's Language'. American Psychologist, 29, 103-114. doi.org/10.1037/h0036026

Tomasello, M. (2004). 'Syntax or Semantics? Response to Lidz et al.' Cognition, 93, 139-140. doi.org/10.1016/j.cognition.2003.09.015

Trafton, A. (2014, 15 September). 'Neuroscientists Identify Key Role of Language Gene', MIT News. Available from newsoffice.mit.edu/2014/language-gene-0915. 
This text is taken from The ANU Undergraduate Research Journal, Volume Eight, 2016, edited by Daniel McKay, published 2017 by ANU eView, The Australian National University, Canberra, Australia.

dx.doi.org/10.22459/AURJ.08.2016.14 